\title{
EGR1 regulates radiation-induced apoptosis in head and neck squamous cell carcinoma
}

\author{
TAE MI YOON ${ }^{1}$, SUN-AE KIM ${ }^{1}$, DONG HOON LEE ${ }^{1}$, JOON KYOO LEE ${ }^{1}$, YOUNG-LAN PARK ${ }^{2}$, \\ KYUNG-HWA LEE ${ }^{3}$, IK-JOO CHUNG ${ }^{2}$, YOUNG-EUN JOO ${ }^{2}$ and SANG CHUL LIM ${ }^{1}$ \\ Departments of ${ }^{1}$ Otorhinolaryngology-Head and Neck Surgery, ${ }^{2}$ Internal Medicine and ${ }^{3}$ Pathology, \\ Chonnam National University Medical School, Gwangju, Republic of Korea
}

Received October 7, 2014; Accepted December 22, 2014

DOI: $10.3892 /$ or.2015.3747

\begin{abstract}
The transcription factor, early growth response 1 (EGR1) belongs to the early growth response family. EGR1 regulates the transactivation of genes involved in growth inhibition and apoptosis by ionizing radiation. The aims of the present study were to evaluate the expression of EGR1, and its relationship to prognosis, in patients with advanced laryngeal and hypopharyngeal squamous cell carcinoma (LHSCC) receiving chemoradiation therapy, and to observe the effect of EGR1 on the apoptosis of head and neck squamous cell carcinoma (HNSCC) cells treated with ionizing radiation. Expression of the EGR1 protein in tissue samples from patients with LHSCC was detected by immunohistochemistry. A high expression of the EGR1 protein was observed in $37(67.3 \%)$ of the 55 LHSCC tissue samples examined. A high EGR1 protein expression in patients with LHSCC who were treated with chemoradiation was significantly associated with improved larynx-preservation survival $(\mathrm{p}=0.04)$. The 5 -year diseasespecific survival rate with larynx preservation was $59 \%$ in patients with a high EGR1 protein expression vs. $30 \%$ in those with a low EGR1 protein expression. In the human HNSCC cell line, PCI50, EGR1 mRNA expression was induced at 30-60 min, and EGR1 protein expression was induced at 60-120 $\mathrm{min}$, after exposure to a 5 Gy dose of ionizing radiation. To evaluate the impact of EGR1 on radiation-induced apoptosis, we used small-interfering RNA to knock down endogenous EGR1 gene expression. Cleaved caspase 3, cleaved caspase 7, and cleaved PARP were decreased, while XIAP was increased, in EGR1-knockdown PCI50 cells compared to negative control PCI50 cells, at all observed post-irradiation time points. These findings suggested that EGR1 knockdown inhibits radiation-induced apoptosis. In conclusion, EGR1
\end{abstract}

Correspondence to: Dr Sang Chul Lim, Department of Otorhinolaryngology-Head and Neck Surgery, Chonnam National University Medical School, 8 Hak-Dong, Dong-Ku, Gwangju 501-757, Republic of Korea

E-mail: limsc@chonnam.ac.kr

Key words: EGR1 protein, radiotherapy, apoptosis, head and neck squamous cell carcinoma may be associated with larynx-preservation survival, through the regulation of radiation-induced apoptosis in patients with LHSCC treated with chemoradiation. Although further investigations are required to support the present study, EGR1 serves as a favorable biomarker of radiosensitivity in the treatment of LHSCC.

\section{Introduction}

The ongoing treatment of head and neck squamous cell carcinoma (HNSCC) is currently in the era of chemoradiation. The advanced cancer stage at diagnosis and aggressive tumor biology of HNSCC contribute to a poor prognosis, with 5-year survival rates ranging from 10 to $40 \%$ (1). In the past, treatment of advanced laryngohypopharyngeal squamous cell carcinoma (LHSCC) predominantly focused on cure by comprehensive surgery, and involved total laryngectomy or laryngopharyngectomy, with consideration given to adjuvant radiotherapy $(2,3)$. Although this approach produces high rates of local tumor control, it affects laryngeal function, resulting in a permanent tracheostomy, and has a negative effect on a patient's quality of life, with consequences such as social isolation $(4,5)$. These are the reasons for the current era of chemoradiation in the treatment of LHSCC. Many patients select chemoradiation rather than wide-margin curative surgery with associated functional loss, despite an advanced stage of LHSCC, and appropriate patient selection is necessary in order to improve the outcome of chemoradiation therapy. Therefore, otolaryngologists have paid increasing attention to specific biomarkers of radiation resistance or sensitivity in HNSCC.

Ionizing radiation exposure is associated with the activation of certain immediate early genes that encode transcription factors, including those of the JUN, FOS and early growth response (EGR) families (6). EGRl, a member of the EGR family, is a pivotal gene that initiates early signal transduction events in response to ionizing radiation, leading to either growth inhibition or apoptosis in tumor cells (6). In human melanoma cells, exposure to ionizing radiation caused induction of the EGR1 protein, whereas inhibition of EGR1 protein expression resulted in a reduced growth-inhibitory response to ionizing radiation (7). Among a group of patients with EGR1overexpressing esophageal squamous cell carcinoma, those who underwent radiotherapy had an improved prognosis (8). 
Findings of the above studies suggested that EGR1 is associated with radiosensitivity in human types of cancer. However, the expression and functional role of EGR1 in response to ionizing radiation has not been elucidated in the LHSCC treatment.

In the present study, we examined EGRl gene expression, and evaluated the relationship between its expression and clinicopathological characteristics of LHSCC, in a welldefined series of patients with advanced cancers and receiving chemoradiation therapy. Additionally, in order to explore the role of EGR1 in ionizing radiation-induced apoptosis, we investigated the induction of EGR1 by ionizing radiation, and determined whether this affected the cleavage or expression of apoptosis-associated proteins in a human LHSCC cell line. To the best of our knowledge, the present study is the first to demonstrate the role of EGR1 in the radiosensitivity of LHSCC.

\section{Materials and methods}

Patients and tumor specimens. To evaluate the expression of the EGR1 protein, sections of paraffin-embedded tissue samples were analyzed from 55 patients who underwent diagnostic biopsy for advanced LHSCC (stage III and IV) at the Chonnam National University Hwasun Hospital (Jeonnam, Korea) between July, 2004 and February, 2009. A total of 60 patients were treated with three cycles of combined cisplatin-, 5-fluorouracil- and docetaxel-based induction chemotherapy (IC). This was followed by cisplatin-based concurrent chemoradiation therapy in 56 patients who showed a complete response (CR) or partial response (PR) after IC, and in two patients who declined total laryngectomy salvage therapy, despite having stable disease (SD) after IC, while another two patients with SD after IC underwent total laryngectomy salvage therapy. Five of the initial 60 patients were subsequently excluded from the present study due to exhaustion of the small block of paraffin-embedded tissue sample taken for the diagnostic biopsy analysis. CR was defined as no visible or palpable disease, as assessed by physical examination, laryngoscopy, and computed tomography. PR was defined as a $>50 \%$ decrease in tumor size, compared with the measurement prior to treatment. SD was defined as stationary or progressive disease. Patient clinicopathological characteristics were reviewed in hospital records. Tumors were staged according to the 7th edition of the American Joint Committee on Cancer Staging Manual (9). Patient survival was measured from the initiation date of the chemotherapy to the date of death or the date of last follow-up. The present study was approved by the Institutional Review Board of Chonnam National University Hwasun Hospital.

Immunohistochemistry. Paraffin-embedded tissue sections were deparaffinized and rehydrated. The tissue sections were incubated with a polyclonal rabbit anti-human EGR1 antibody (Santa Cruz Biotechnology, Inc., Santa Cruz, CA, USA), and then stained using the Dako Real ${ }^{\mathrm{TM}}$ EnVision $^{\mathrm{TM}}$ horseradish peroxidase (HRP)-3,3'-diaminobenzidine detection system (Agilent Technologies, Santa Clara, CA, USA). Two independent observers assessed the staining intensity of each specimen, without any knowledge of the clinical information. The staining intensity was scored as follows: 0 , no staining of tumor cells; $1+$ weak or comparable staining in the cytoplasm and/or nucleus compared to that of non-tumor cells; $2+$, readily appreciable dark-brown staining, delineating the tumor cell cytoplasm and/ or nucleus. A score of 0 or 1+ was regarded as a low expression and $2+$ as a high expression of the EGR1 protein.

Cell culture and transfection. The human PCI50 HNSCC cell line was provided by Dr M.W. Sung (Seoul National University, Seoul, South Korea). The cells were cultured in RPMI-1640 medium (Invitrogen, Carlsbad, CA, USA), supplemented with $10 \%$ fetal bovine serum (FBS) (HyClone Laboratories, Logan, UT, USA), in a humidified atmosphere of $5 \% \mathrm{CO}_{2}$ at $37^{\circ} \mathrm{C}$. Small-interfering RNA (siRNA) was used to knock down endogenous EGRl gene expression in PCI50 cells. The cells were transfected for $48 \mathrm{~h}$ with EGR1-specific siRNA (Santa Cruz Biotechnology, Inc.) or negative control siRNA (Qiagen, Germantown, MD, USA) using Lipofectamine 2000 (Invitrogen).

Cell irradiation. The cells were irradiated with a $6 \mathrm{MV}$ photon beam delivered by a linear accelerator. Water-equivalent calibration plates of $1-\mathrm{cm}$ thickness were placed above and below the culture dish. The attached cells in the bottom of the culture dish were covered to a $5-\mathrm{mm}$ depth by culture medium.

Protein isolation and western blot analysis. The cells were lysed in RIPA buffer (1 M Tris $\mathrm{HCl}, 150 \mathrm{mM} \mathrm{NaCl}, 1 \%$ Triton X-100,2 mMEDTA) with $1 \mathrm{mM}$ phenylmethanesulfonyl fluoride, Halt ${ }^{\mathrm{TM}}$ phosphatase inhibitor, and Halt ${ }^{\mathrm{TM}}$ protease inhibitor cocktail (Thermo Fisher Scientific, Rockford, IL, USA). Proteins (10-20 $\mu \mathrm{g}$ ) were resolved by electrophoresis, and transferred to polyvinylidene difluoride membranes (EMD; Millipore, Billerica, MA, USA). Specific proteins were detected by sequentially probing blots with cognate primary antibodies: EGR1 (Santa Cruz Biotechnology, Inc.), cleaved caspase 3, cleaved caspase 7, cleaved poly(ADP-ribose) polymerase (PARP), the X-linked inhibitor of apoptosis protein (XIAP) (Cell Signaling Technology, Danvers, MA, USA), and glyceraldehyde 3-phosphate dehydrogenase (GAPDH; Santa Cruz Biotechnology, Inc.). Immunoreactive proteins were visualized using an enhanced chemiluminescence detection system with an HRP substrate (EMD), and analyzed using the LAS-4000 luminescent image analyzer (Fujifilm, Tokyo, Japan).

RNA isolation and reverse transcription polymerase chain reaction (RT-PCR). Total RNA was extracted from cells using TRIzol ${ }^{\circledR}$ reagent (Invitrogen), reverse transcribed and amplified using specific primers for EGR1 and GAPDH. The primer sequences used were: EGR1, 5'-TCGCCTCGATGGAGC TCCTC-3'/5'-CATGTGTGCCACTGTGACGT-3'; and GAPDH, 5'-ACCACAGTCCATGCCATC AC-3'/5'-TCCACCA CCCTGTTGCTGTA-3'. For cDNA synthesis, $1 \mu \mathrm{g}$ mRNA was incubated with $50 \mathrm{ng} / \mu 1$ oligo(dT) (Promega, Madison, WI, USA), Moloney murine leukemia virus reverse transcriptase (Invitrogen), and RNAsin (Takara Bio, Shiga, Japan). PCR amplification of cDNA was carried out using specific primers (see above) and GoTaq ${ }^{\circledR}$ DNA polymerase (Promega). PCR products were separated by electrophoresis in a $1 \%$ agarose gel containing ethidium bromide. 


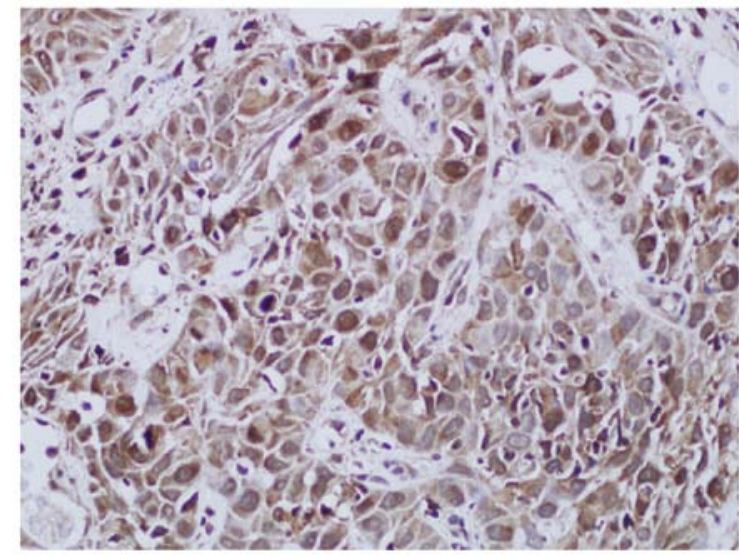

Figure 1. Immunohistochemical staining of EGR1 in biopsy tissue from laryngeal and hypopharyngeal squamous cell carcinoma patients. LHSCC cells in the tissue sample showed 2+ EGR1 immunostaining, indicating high EGR1 expression. Magnification, x200. EGR1, early growth response 1; LHSCC, laryngeal and hypopharyngeal squamous cell carcinoma.

Statistical analyses. The relationship between EGR1 protein expression and various clinicopathological parameters was compared using the $\chi^{2}$ and Fisher's exact tests. Survival curves were calculated using the Kaplan-Meier method, and comparison of curves was conducted using the log-rank test. The Statistical Package for the Social Sciences, version 21.0 (MicroCal Software Inc., Chicago, IL, USA) was used for all analyses. $\mathrm{P}<0.05$ was considered to indicate a statistically significant result.

Ethical considerations. Local research ethics committee approval from the Chonnam National University Hwasun Hospital Institutional Review Board.

\section{Results}

Expression of EGRl is increased in human LHSCC tissue. The patients in the present study included 54 men and one woman. The mean age was $64.4 \pm 8.0$ years (mean \pm standard deviation), with a range of $47-83$ years. The mean follow-up period while the patient was alive was $49.9 \pm 30.17$ months with a range of 8.1-121.3 months. To decrease the influence due to variable treatment strategies and evaluate whether EGR1 contributes to the radiosensitivity of LHSCC, only patients who were treated with the same chemoradiation regimen were included in the present study.

EGR1 protein expression was investigated by immunohistochemical staining of formalin-fixed, paraffin-embedded biopsy tissue obtained from 55 LHSCC patients. Immunohistochemical staining revealed the overexpression of EGR1 in LHSCC tissue (Fig. 1). Based on our criteria, a high expression of EGR1, with $2+$ staining, was observed in $67.3 \%$ of patients $(37 / 55)$, while a low expression of EGR1, with 1+ or no staining, was observed in $32.7 \%$ of patients $(18 / 55)$.

High expression of EGRl is significantly associated with improved survival with larynx preservation in patients with LHSCC treated with chemoradiation. Patient data, and the correlation between EGR1 expression and various clinicopathological factors in LHSCC, are shown in Table I.
Table I. Correlation between EGR1 expression and clinicopathological parameters in patients with laryngeal and hypopharyngeal squamous cell carcinoma.

\begin{tabular}{|c|c|c|c|c|}
\hline \multirow[b]{2}{*}{ Parameters } & \multirow[b]{2}{*}{$\begin{array}{l}\text { Total } \\
(\mathrm{n}=55)\end{array}$} & \multicolumn{2}{|c|}{ EGR1 expression } & \multirow[b]{2}{*}{ P-value } \\
\hline & & $\begin{array}{c}\text { Low } \\
(n=18)\end{array}$ & $\begin{array}{l}\text { High } \\
(n=37)\end{array}$ & \\
\hline Age (years) & & & & 0.39 \\
\hline$<65$ & 27 & 7 & 20 & \\
\hline$\geq 65$ & 28 & 11 & 17 & \\
\hline Gender & & & & 1.00 \\
\hline Male & 54 & 18 & 36 & \\
\hline Female & 1 & 0 & 1 & \\
\hline Location & & & & 1.00 \\
\hline Larynx & 32 & 11 & 21 & \\
\hline Hypopharynx & 23 & 7 & 16 & \\
\hline Stage & & & & 1.00 \\
\hline III & 20 & 6 & 14 & \\
\hline IV & 35 & 12 & 23 & \\
\hline T stage & & & & 0.17 \\
\hline $\mathrm{T} 1, \mathrm{~T} 2$ & 26 & 6 & 20 & \\
\hline $\mathrm{T} 3, \mathrm{~T} 4$ & 29 & 12 & 17 & \\
\hline N stage & & & & 0.57 \\
\hline N0, N1 & 27 & 10 & 17 & \\
\hline $\mathrm{N} 2$ & 28 & 8 & 20 & \\
\hline $\begin{array}{l}\text { Tumor response } \\
\text { after IC }\end{array}$ & & & & 1.00 \\
\hline $\mathrm{CR}, \mathrm{PR}$ & 51 & 17 & 34 & \\
\hline $\mathrm{SD}$ & 4 & 1 & 3 & \\
\hline $\begin{array}{l}\text { Overall tumor } \\
\text { response after CRT }\end{array}$ & & & & 0.30 \\
\hline $\mathrm{CR}$ & 42 & 16 & 26 & \\
\hline $\mathrm{PR}, \mathrm{SD}$ & 11 & 2 & 9 & \\
\hline $\begin{array}{l}\text { Disease-specific } \\
\text { survival }\end{array}$ & & & & 0.38 \\
\hline Dead & 22 & 9 & 13 & \\
\hline Survival & 33 & 9 & 24 & \\
\hline $\begin{array}{l}\text { Larynx-preservation } \\
\text { survival }\end{array}$ & & & & $0.04^{\mathrm{a}}$ \\
\hline $\begin{array}{l}\text { Dead or total } \\
\text { laryngectomy }\end{array}$ & 26 & 12 & 14 & \\
\hline Survival & 29 & 6 & 23 & \\
\hline
\end{tabular}

EGR1, early growth response 1; IC, induction chemotherapy; CRT, chemoradiation; CR, complete response; PR, partial response; SD,

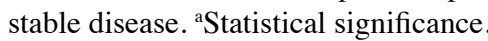

A high EGR1 expression was significantly associated with improved survival with laryngeal preservation $(\mathrm{p}=0.04)$. However, no significant correlation was found between EGR1 expression and other clinicopathological factors, including age, gender, tumor location, tumor stage, $\mathrm{T}$ stage (tumor 


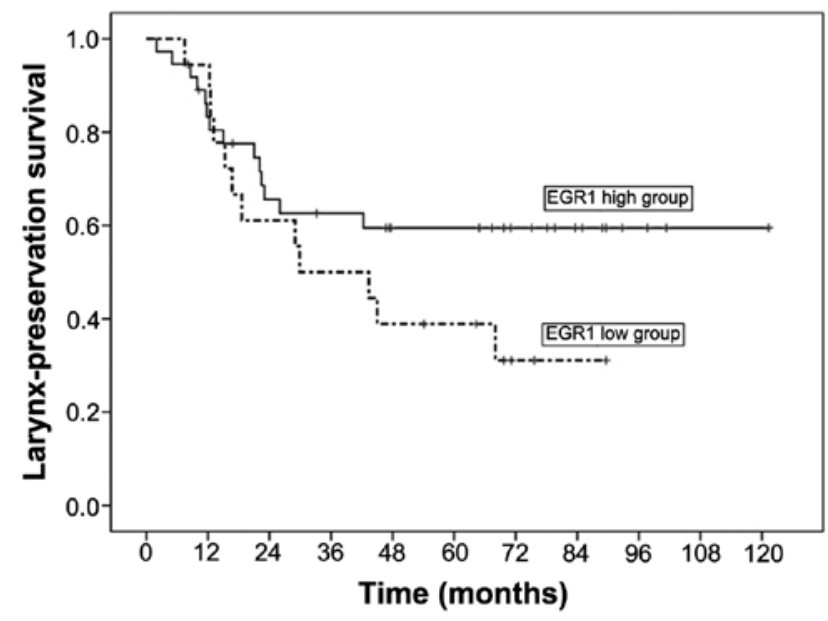

Figure 2. Kaplan-Meier curves show survival with larynx preservation of patients with laryngeal and hypopharyngeal squamous cell carcinoma in relation to EGR1 expression. Solid line, patients with high EGR1 expression; dotted line, patients with low EGR1 expression. EGR1, early growth response 1 .
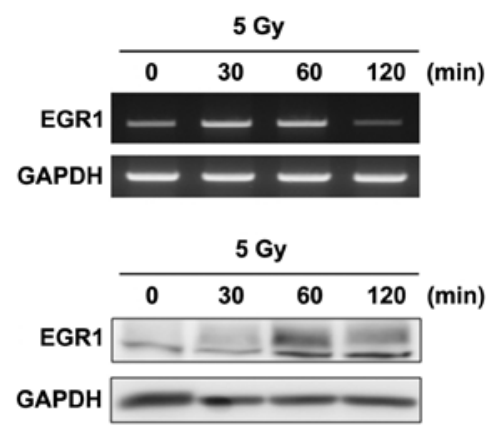

Figure 3. EGR1 mRNA and EGR1 protein expression following irradiation of head and neck squamous cell carcinoma cells. PCI50 cells were irradiated with a 5 Gy dose. EGR1 mRNA expression was increased at 30-60 min, and EGR1 protein expression at 60-120 min, post-irradiation. EGR1, early growth response 1 .

invasion), $\mathrm{N}$ stage (lymph-node metastasis), chemotherapy sensitivity, tumor response following chemoradiation therapy and disease-specific survival ( $p>0.05)$.

For the 55 patients with stage III or IV LHSCC that were enrolled in the present study, the 3-/5-year disease-specific survival rate was $60 / 55 \%$, and the 3-/5-year disease-specific survival rate with larynx preservation was $52 / 49 \%$. The 5 -year disease-specific survival rate with larynx preservation was 59\% in patients with high EGR1 expression vs. $30 \%$ in patients with low EGR1 expression, however, analysis of Kaplan-Meier curves for survival with larynx preservation, using the log-rank test, did not demonstrate a significant difference between these two groups of patients ( $\mathrm{p}=0.15$ ) (Fig. 2).

Radiation upregulates the EGRl, and knockdown of EGRI inhibits radiation-induced apoptosis in human HNSCC cells. To determine whether radiation in upregulation of the EGR1, total RNA and whole-cell protein extracts were prepared from PCI50 HNSCC cells at different time intervals following exposure to a $5 \mathrm{~Gy}$ dose of ionizing radiation, and analyzed by RT-PCR and western blotting, respectively. As shown in
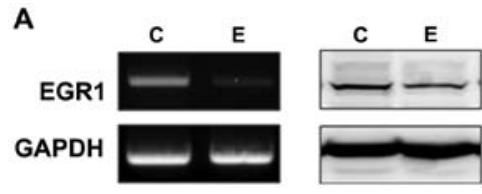

B

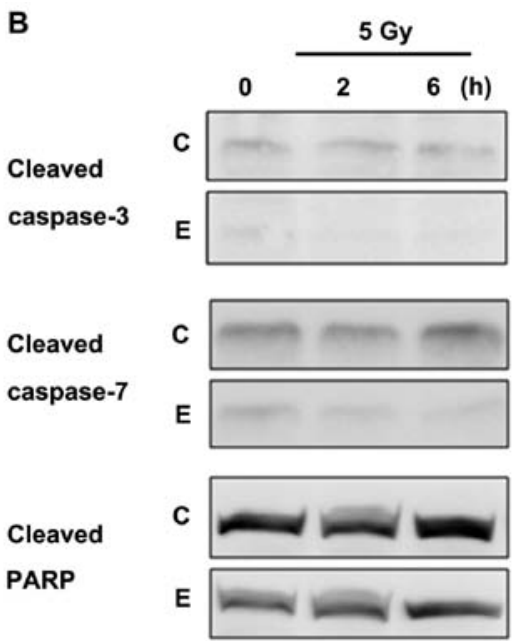

XIAP

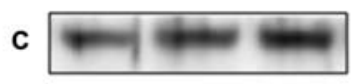

$\mathrm{E}$

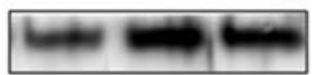

GAPDH

c

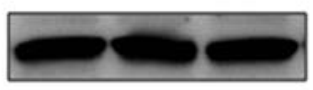

E

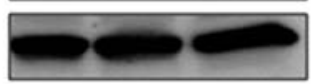

Figure 4. Effect of EGR1 knockdown on radiation-induced apoptosis in head and neck squamous cell carcinoma cells. (A) Expression of EGR1 mRNA and EGR1 protein is decreased by siRNA-mediated EGR1 knockdown in PCI-50 cells.(B) Level of cleaved caspase 3, cleaved caspase 7 and cleaved PARP was decreased, while the level of XIAP was increased, in the EGR1 knockdown cells (E), as compared to the negative control cells (C), at all time points. EGR1, early growth response 1 .

Fig. 3, EGR1 mRNA expression was induced at 30-60 min post-irradiation and EGR1 protein expression was induced at 60-120 min post-irradiation.

To evaluate the impact of EGR1 on radiation-induced apoptosis, we used siRNA to inhibit endogenous EGRl gene expression. EGR1 mRNA and EGR1 protein expression were reduced by EGR1-specific siRNA, as compared to the negative control siRNA, in transfected PCI50 cells (Fig. 4A). The levels of the cleaved caspase 7, cleaved PARP and XIAP, were increased in the negative control siRNA-transfected PCI50 cells at $6 \mathrm{~h}$ after a $5 \mathrm{~Gy}$ irradiation dose, as compared to the levels at $0 \mathrm{~h}$ (Fig. 4B). By contrast, the levels of cleaved caspase 3, cleaved caspase 7, and cleaved PARP were decreased, while the level of XIAP was increased, in EGR1-specific siRNA-transfected PCI50 cells, as compared to the negative control cells, at all time points (Fig. 4B). These findings suggested that EGR1 knockdown inhibits radiation-induced apoptosis.

\section{Discussion}

A combination of radiotherapy and molecular-targeted therapy, based on an understanding of the molecular changes 
that underline the development of cancer, is one of the most promising treatment strategies (10). Treatment strategies such as chemotherapy and radiotherapy eliminate cancer cells by the induction of apoptosis, as well as mitotic death. Therefore, the induction of apoptosis in tumor cells is important in the efficacy of radiation therapy and chemotherapy (11). The EGRI gene is crucial for the initiation of early signal transduction in response to radiation, and controls apoptosis in tumor cells (6). EGR 1 is one of the candidate genes whose expression is likely to be targeted by radiotherapy.

The EGR family includes EGR1, EGR2, EGR3, EGR4 and the tumor suppressor and Wilms' tumor gene product, WT1 (12). EGR1 is a nuclear protein that contains three zinc fingers of the $\mathrm{C}_{2} \mathrm{H}_{2}$ subtype (13). The amino acids constituting the zinc finger motif confer DNA-binding function, whereas the NH2-terminal amino acids confer transactivation function, on the EGR1 structure (13). EGR1 is induced by many different stimuli, ranging from growth factors and cytokines to stress signals, such as ultraviolet and ionizing radiation, apoptosis-promoting factors and injury (14). Notably, depending on the cell type, EGR1 may act as a positive or negative regulator of gene transcription (13). In tumor cell lines and tumors representing prostate, kidney and gastric cancer, EGR1 stimulated tumor cell growth and was associated with a poor prognosis $(15,16)$. By contrast, in fibrosarcoma, glioblastoma, melanoma and esophageal lung and breast cancer, EGR1 is reported to be a tumor suppressor $(7,8,17-20)$. Of the head and neck cancers, only in nasopharyngeal carcinoma was it found that the expression of EGR1 had a positive prognostic effect on survival (21). The functional role of EGR1 in LHSCC has not been reported.

In the present study, to examine the role of EGR1 in the response to ionizing radiation, we evaluated the relationship between its expression and other variables, in patients with advanced LHSCC receiving chemoradiation therapy. Our study results show that a high expression of EGR1 was significantly associated with improved survival with larynx preservation. The 5-year disease-specific survival rate with larynx preservation was $59 \%$ in patients with a high EGR1 expression vs. $30 \%$ in patients with a low EGR1 expression. This finding is important because survival with larynx preservation is one of the important end points for evaluating the efficacy of a treatment strategy for LHSCC. However, contrary to our expectations, the CR rate and analysis of survival curves using the Kaplan-Meier method did not demonstrate a significant difference between the two groups of patients with high vs. low EGR1 expression. It is possible that the survival rate of patients with LHSCC can be affected by many factors, and the number of LHSCC patients in the present study was not sufficiently large to provide the additional insights necessary to identify the important factors. However, our results suggest that EGR1 expression serves as a favorable biomarker of radiosensitivity in human LHSCC patients receiving chemoradiation.

We also investigated the role of EGR1 in ionizing radiation-induced apoptosis in the human PCI50 HNSCC cell line. EGR1 mRNA and EGR1 protein expression was induced at 30-120 min post-irradiation in PCI50 cells. The siRNA-mediated EGR1 knockdown in PCI50 cells resulted in decreased levels of cleaved caspase 3 , cleaved caspase 7 , and cleaved PARP, which are key enzymes involved in apoptosis. These findings suggest that EGR1 knockdown inhibits radiation-induced apoptosis. Our results are in agreement with those of previous reports. Ahmed et al reported that EGR1-blocked melanoma cells showed significantly reduced $(<50 \%)$ sensitivity to radiation-inducible growth inhibition, and this resistance was dose-dependent (7). Das et al reported that EGR1 induction is involved in the regulation of radiationinducible apoptosis, despite the presence of wild-type p53, in primary mouse embryonic fibroblasts (22). The results of our study and those of previous reports suggest that EGR1 may be associated with radiosensitivity.

The present study has two limitations. Firstly, the sample size of patients with LHSCC receiving chemoradiation was not large enough to reach a significant conclusion, although it is still valuable as a rare LHSCC study with homogenous treatment groups. Secondly, only a single HNSCC cell line was used. Despite these limitations, the present study is the first to demonstrate the role of EGR1 in the radiosensitivity of LHSCC.

In conclusion, EGR1 may be associated with survival with larynx preservation through the regulation of radiation-induced apoptosis in patients with LHSCC treated with chemoradiation. Although further investigations are required to support the present study, EGR1 serves as a favorable biomarker of radiosensitivity in the treatment of human LHSCC.

\section{Acknowledgements}

The present study was supported by a grant (CRI 12018-1) Chonnam National University Hospital Research Institute of Clinical Medicine. We thank Dr M.W. Sung (Seoul National University) for the PCI50 cell line.

\section{References}

1. Goldberg HI, Lockwood SA, Wyatt SW and Crossett LS: Trends and differentials in mortality from cancers of the oral cavity and pharynx in the United States, 1973-1987. Cancer 74: 565-572, 1994.

2. Bussu F, Miccichè F, Rigante M, et al: Oncologic outcomes in advanced laryngeal squamous cell carcinomas treated with different modalities in a single institution: a retrospective analysis of 65 cases. Head Neck 34: 573-579, 2012.

3. Takes RP, Strojan P, Silver CE, et al: Current trends in initial management of hypopharyngeal cancer: the declining use of open surgery. Head Neck 34: 270-281, 2012.

4. American Society of Clinical Oncology, Pfister DG, Laurie SA, et al: American Society of Clinical Oncology clinical practice guideline for the use of larynx-preservation strategies in the treatment of laryngeal cancer. J Clin Oncol 24: 3693-3704, 2006.

5. Fung K, Lyden TH, Lee J, et al: Voice and swallowing outcomes of an organ-preservation trial for advanced laryngeal cancer. Int J Radiat Oncol Biol Phys 63: 1395-1399, 2005.

6. Ahmed MM: Regulation of radiation-induced apoptosis by early growth response-1 gene in solid tumors. Curr Cancer Drug Targets 4: 43-52, 2004.

7. Ahmed MM, Venkatasubbarao K, Fruitwala SM, et al: EGR-1 induction is required for maximal radiosensitivity in A375-C6 melanoma cells. J Biol Chem 271: 29231-29237, 1996.

8. Wu MY, Wu XY, Li QS and Zheng RM: Expression of Egr-1 gene and its correlation with the oncogene proteins in nonirradiated and irradiated esophageal squamous cell carcinoma. Dis Esophagus 19: 267-272, 2006.

9. American Joint Committee on Cancer Staging Manual. 7th edition. Springer, 2010.

10. Mezhir JJ, Smith KD, Posner MC, et al: Ionizing radiation: a genetic switch for cancer therapy. Cancer Gene Ther 13: 1-6, 2006. 
11. Dey S, Spring PM, Arnold S, et al: Low-dose fractionated radiation potentiates the effects of Paclitaxel in wild-type and mutant p53 head and neck tumor cell lines. Clin Cancer Res 9: 1557-1565, 2003.

12. Rose EA, Glaser T, Jones C, et al: Complete physical map of the WAGR region of $11 \mathrm{p} 13$ localizes a candidate Wilms' tumor gene. Cell 60: 495-508, 1990.

13. Gashler AL, Swaminathan S and Sukhatme VP: A novel repression module, an extensive activation domain, and a bipartite nuclear localization signal defined in the immediate-early transcription factor Egr-1. Mol Cell Biol 13: 4556-4571, 1993.

14. Fahmy RG and Khachigian LM: Antisense Egr-1 RNA driven by the CMV promoter is an inhibitor of vascular smooth muscle cell proliferation and regrowth after injury. J Cell Biochem 84: 575-582, 2002.

15. Adamson ED and Mercola D: Egrl transcription factor: multiple roles in prostate tumor cell growth and survival. Tumour Biol 23: 93-102, 2002.

16. Myung E, Park YL, Kim N, et al: Expression of early growth response-1 in human gastric cancer and its relationship with tumor cell behaviors and prognosis. Pathol Res Pract 209: 692-699, 2013.

17. Liu C, Yao J, de Belle I, Huang RP, Adamson E and Mercola D: The transcription factor EGR-1 suppresses transformation of human fibrosarcoma HT1080 cells by coordinated induction of transforming growth factor- $\beta 1$, fibronectin, and plasminogen activator inhibitor-1. J Biol Chem 274: 4400-4411, 1999.
18. Liu C, Yao J, Mercola D and Adamson E: The transcription factor EGR-1 directly transactivates the fibronectin gene and enhances attachment of human glioblastoma cell line U251. J Biol Chem 275: 20315-20323, 2000.

19. Huang RP, Fan Y, de Belle I, et al: Decreased Egr-1 expression in human, mouse and rat mammary cells and tissues correlates with tumor formation. Int J Cancer 72: 102-109, 1997.

20. Shareef MM, Cui N, Burikhanov R, et al: Role of tumor necrosis factor- $\alpha$ and TRAIL in high-dose radiation-induced bystander signaling in lung adenocarcinoma. Cancer Res 24: 11811-11820, 2007.

21. Kim YJ, Go H, Wu HG, Jeon YK, Park SW and Lee SH: Immunohistochemical study identifying prognostic biomolecular markers in nasopharyngeal carcinoma treated by radiotherapy. Head Neck 33: 1458-1466, 2011.

22. Das A, Chendil D, Dey S, et al: Ionizing radiation down-regulates p53 protein in primary Egr-1/- mouse embryonic fibroblast cells causing enhanced resistance to apoptosis. J Biol Chem 276: 3279-3286, 2001. 